\title{
HARMONIC TWO-FORMS IN FOUR DIMENSIONS
}

\author{
WALTER SEAMAN
}

(Communicated by Jonathan M. Rosenberg)

\begin{abstract}
Conformal invariance of middle-dimensional harmonic forms is used to improve Kato's inequality for four-manifolds. An application to positively curved four-manifolds is given.
\end{abstract}

\section{INTRODUCTION}

The purpose of this paper is to prove the following:

Theorem 1. Let $\left(M^{4}, g\right)$ be a four-dimensional Riemannian manifold. Let $\omega$ be a harmonic two-form on $(M, g)$. Then $\omega$ satisfies the pointwise inequality:

$$
|\nabla \omega|^{2} \geq\left.\frac{3}{2}|d| \omega\right|^{2} \text {. }
$$

Kato's inequality [1, p. 130], states that if $E$ is a Riemannian vector bundle with connection $\bar{\nabla}$ over a Riemannian manifold $M$, then any smooth section $s$ of $E$, satisfies the pointwise inequality:

$$
|\overline{\nabla s}|^{2} \geq|d| s||^{2} .
$$

Now by definition, if $s(\omega)$ vanishes at $p \in M$, then $d|s|(d|\omega|)=0$ at $p$. Thus, $(0.1)$ and $(0.2)$ are automatically valid at such a point. At points where $\omega$ does not vanish $(0.1)$ can be thought of as a quantitative improvement of (0.2), for the case of harmonic two-forms on four-dimensional manifolds.

As an application of the above theorem, we prove:

Theorem 2. Let $\left(M^{4}, g\right)$ be a compact, connected four-dimensional Riemannian manifold whose sectional curvature $K(g)$ satisfies $1 \geq K(g) \geq \delta$. If

$$
\delta \geq 1 /\left(3\left(1+3 \cdot 2^{1 / 4} / 5^{1 / 2}\right)^{1 / 2}+1\right) \approx .1714
$$

then $M$ is definite.

This theorem represents an improvement of results starting with [2] followed by $[4,7,6]$. The relevance of Theorems 1 and 2 stems from the following facts

Received by the editors November 6, 1989 and, in revised form, July 27, 1990.

1980 Mathematics Subject Classification (1985 Revision). Primary 53C20; Secondary 57N13.

Key words and phrases. Harmonic forms, Weitzenbock operator, curvature, four-manifolds. 
(cf. $[2,4,7,6]):$ the "Sphere Theorem" classifies compact simply-connected Riemannian manifolds whose sectional curvature $K$ satisfies $1 \geq K \geq \frac{1}{4}$, while the Hopf conjecture states that $\mathbb{S}^{2} \times \mathbb{S}^{2}$ does not admit a strictly positively curved metric. In Theorem 2, the curvature pinching is below $\frac{1}{4}$, and the resulting $M$ must be definite. As $\mathbb{S}^{2} \times \mathbb{S}^{2}$, as well as $\mathbb{S}^{2} \times \mathbb{S}^{2} \sharp \mathbb{C} P^{2} \sharp \cdots \sharp \mathbb{C} P^{2} \sharp \overline{\mathbb{C} P^{2}} \sharp \cdots \sharp \overline{\mathbb{C} P^{2}}$ (connected sum, where $\overline{\mathbb{C} P^{2}}$ means $\mathbb{C} P^{2}$ with the usual orientation reversed) are all indefinite, these manifolds thus cannot admit metrics with sectional curvature so pinched as to satisfy 0.3 . At this point, we are not able to prove that an $M$ satisfying the above hypotheses is topologically $\mathbb{S}^{4}$ or $\mathbb{C} P^{2}$, since the arguments in [7] do not appear to extend down to the pinching in Theorem 2.

The idea behind Theorem 1 is as follows: let $M_{0}$ be the open subset of $M$ where $\omega$ does not vanish. Change $g$ conformally to a new metric $g^{\prime}$ on $M_{0}$ relative to which $\omega$ has constant length. By the conformal invariance of middle-dimensional harmonic forms, $\omega$ is still harmonic for the Riemannian manifold $\left(M_{0}, g^{\prime}\right)$. Writing out the formula $((1.8) \S 1)$ for $R_{2}^{\prime}$, the Weitzenbock operator on two-forms for $g^{\prime}$, in terms of $R_{2}$, the corresponding operator for $g$, and using the harmonicity of $\omega$ for either metric, proves (0.1) on $M_{0}$. As mentioned, this then proves $(0.1)$ on all of $M$.

\section{BACKGROUND AND PROOFS}

Because there are many notational and sign differences in use for various geometric objects, we will first establish the notation to be used in this paper.

If $(M, g)$ is a Riemannian manifold, with Riemannian connection $\nabla$, then the curvature tensor, $R$ considered as a $(3,1)$ tensor, is given by

$$
R(X, Y) Z=\nabla_{X} \nabla_{Y} Z-\nabla_{Y} \nabla_{X} Z-\nabla_{[X, Y]} Z,
$$

where $X, Y, Z$ are vector fields on $M$. For a plane $P$ spanned by (orthonormal) $X_{p}, Y_{p} \in T_{p} M$, the sectional curvature of $P$ is given by

$$
K(P)=g_{p}(R(X, Y) Y, X)=\langle R(X, Y) Y, X\rangle_{p} .
$$

Aside from (1.1) and (1.2), our conventions are the same as in [3].

If $f$ is a smooth function on $M$, and $g^{\prime}$ is the metric given by $g^{\prime}=e^{2 f} g$, then, considered as a $(4,0)$ tensor, we have, for $R^{\prime}$, the curvature tensor of $g^{\prime}$ :

$$
R^{\prime}=e^{2 f}\left(R+g \otimes\left(D d f-d f \circ d f+\frac{1}{2}|d f|^{2} g\right)\right)
$$

(cf. [3, p. 58], the sign difference comes from (1.1), but our Ricci tensors are the same). The Weitzenbock operator $R_{2}$ of $(M, g)$, is the $(4,0)$-tensor given by (cf. [5])

$$
R_{2}=\operatorname{Ric} \oslash g+2 R
$$


That is, at a point $p \in M$, with $v_{i}, w_{i} \in T_{p} M, i=1,2$, we define (1.4.1)

$$
\begin{aligned}
R_{2}\left(v_{1}, v_{2}, w_{1}, v_{2}\right)= & \operatorname{Ric}\left(v_{1}, w_{1}\right) g\left(v_{2}, w_{2}\right)+\operatorname{Ric}\left(v_{2}, w_{2}\right) g\left(v_{1}, w_{1}\right) \\
& -\operatorname{Ric}\left(v_{1}, w_{2}\right) g\left(v_{2}, w_{1}\right)-\operatorname{Ric}\left(v_{2}, w_{1}\right) g\left(v_{1}, w_{2}\right) \\
& +2\left\langle R\left(v_{1}, v_{2}\right) w_{1}, w_{2}\right\rangle .
\end{aligned}
$$

We also take (1.4.1) as the definition of $R_{2}$ considered as a map from twovectors to two-vectors:

$$
\left\langle R_{2}\left(v_{1} \wedge v_{2}\right), w_{1} \wedge w_{2}\right\rangle=R_{2}\left(v_{1}, v_{2}, w_{1}, w_{2}\right) .
$$

Finally, $R_{2}$ is considered as a map from two-forms to two-forms by

$$
R_{2}(\omega)\left(v_{1} \wedge v_{2}\right)=\omega\left(R_{2}\left(v_{1} \wedge v_{2}\right)\right),
$$

where $\omega$ is a two-form at $p$. Considering $R_{2}$ as a map from two-forms to two-forms as in (1.4.3), one has (cf. [6]).

$$
\langle\Delta \omega, \omega\rangle=\frac{1}{2} \Delta|\omega|^{2}+|\nabla \omega|^{2}+\left\langle R_{2} \omega, \omega\right\rangle,
$$

where $|\nabla \omega|^{2}$ is the length of the tensor $\nabla \omega$, and $\langle$,$\rangle denotes the inner$ product induced on two-forms from $g$. For the remainder of the paper, we assume $M$ is four dimensional. In this case, if $\langle,\rangle^{\prime}$ is the inner product on two-forms from $g^{\prime}=e^{2 f} g$, then

$$
\langle,\rangle^{\prime}=e^{-4 f}\langle,\rangle
$$

whereas for the induced inner product on two-vectors, one has

$$
\langle,\rangle^{\prime}=e^{4 f}\langle,\rangle \text {. }
$$

Using (1.3) and (d), p. 59, [3], with $n=4$, we conclude:

$$
R_{2}^{\prime}=e^{2 f}\left[R_{2}+\left(\Delta f-|d f|^{2}\right) g \oslash g\right] \text { as }(4,0) \text {-tensors. }
$$

For $R_{2}$ considered as a map of two-vectors, one has

$$
R_{2}^{\prime}=e^{-2 f}\left(R_{2}+2\left(\Delta f-|d f|^{2}\right) \mathrm{Id}\right) ;
$$

and for $R_{2}$ considered as a map of two-forms the relation between $R_{2}$ and $R_{2}^{\prime}$ is the same as (1.7.1). Using (1.7.1) and (1.6), one finds

$$
\left\langle R_{2}^{\prime} \omega, \omega\right\rangle^{\prime}=e^{-6 f}\left\{\left\langle R_{2} \omega, \omega\right\rangle+2\left(\Delta f-|d f|^{2}\right)|\omega|^{2}\right\},
$$

where $\omega$ is a two-form.

Fix any two-form on $M$, and let $M_{0}$ be the open subset $M_{0}=\left\{p \in M \mid \omega_{p} \neq\right.$ $0\}$. On $M_{0},|\omega|$ is a smooth function. Endow $M_{0}$ with the Riemannian metric

$$
g^{\prime}=|\omega| g, \quad\left(\text { i.e., } e^{2 f}=|\omega|\right)
$$

so $\langle\omega, \omega\rangle^{\prime} \equiv 1$. In this case, one computes that

$$
2\left(\Delta f-|d f|^{2}\right)=\frac{\Delta|\omega|}{|\omega|}+\frac{1}{2} \frac{\left.|d| \omega\right|^{2}}{|\omega|^{2}} \quad\left(\text { on } M_{0}\right)
$$


so, using $\frac{1}{2} \Delta|\omega|^{2}=|\omega| \Delta|\omega|-|d| \omega||^{2}$, we see that (1.8) yields

$$
\left\langle R_{2}^{\prime}, \omega, \omega\right\rangle^{\prime}=|\omega|^{-3}\left\{\left\langle R_{2} \omega, \omega\right\rangle+\frac{1}{2} \Delta|\omega|^{2}+\frac{3}{2}|d| \omega||^{2}\right\} .
$$

Proof of Theorem 1. Let $\omega$ be a harmonic two-form on $(M, g)$. Then $\omega$ is also harmonic on $\left(M_{0}, g\right)$ and, since middle-dimensional harmonic forms are conformally invariant, $\omega$ is harmonic on $\left(M_{0}, g^{\prime}\right)$ with $g^{\prime}$ given by (1.9). Since $\omega$ is $g$ harmonic, (1.5) yields $\left\langle R_{2} \omega, \omega\right\rangle+\frac{1}{2} \Delta|\omega|^{2}=-|\nabla \omega|^{2}$. Since $\omega$ is $g^{\prime}$ harmonic, with constant length, (1.5) also yields $\left\langle R_{2}^{\prime} \omega, \omega\right\rangle^{\prime}=-\left|\nabla^{\prime} \omega\right|^{2}$. Thus (1.10) yields

$$
-\left|\nabla^{\prime} \omega\right|^{\prime 2}=|\omega|^{-3}\left\{-|\nabla \omega|^{2}+\frac{3}{2}|d| \omega||^{2}\right\}
$$

which proves (0.1) on $M_{0}$, and thus on $M$. Q.E.D.

The proof of Theorem 2 follows the same line of argument as [6]. One inserts $(0.1)$ in the arguments of [6] between (4) and (5) to conclude (in the notation of that paper):

$$
\left|\nabla\left(\left|X_{+}\right|-\left|X_{-}\right|\right)\right|^{2} \leq \frac{4}{3}|\nabla X|^{2}
$$

Here $X$ is a two-form on a four-manifold with $X_{+}, X_{-}$the selfdual and antiself-dual components, respectively. Substituting (1.12) into (5) of [6] yields

$$
\int \frac{4}{3}|\nabla X|^{2} \geq \lambda_{1} \int\left(\left|X_{+}\right|-\left|X_{-}\right|\right)^{2}
$$

and the remainder of the proof proceeds as in that paper.

\section{REFERENCES}

1. P. Bérard, Spectral geometry: Direct and inverse problems (with an appendix by G. Besson), Lecture Notes in Math., vol. 1207, Springer-Verlag, 1986.

2. M. Berger, Sur les variétés $\frac{4}{23}$-pincées de dimension 5, C. R. Acad. Sci. Paris Sér. I Math. 257 (1963).

3. A. Besse, Einstein manifolds, Ergeb. Math. Grenzgeb. (3), Folge, Band 10, Springer-Verlag, 1987.

4. J. P. Bourguignon, La conjecture de Hopf sur $\mathbb{S}^{2} \times \mathbb{S}^{2}$, Géometrie riemannienne en dimension 4, Séminaire Arthur Besse, CEDIC Paris, 1981, pp. 347-355.

5. W. Seaman, Two forms on four manifolds, Proc. Amer. Math. Soc. 101 (1987), 353-357.

6. ___ A pinching theorem for four manifolds, Geom. Dedicata 31 (1989), 37-40.

7. M. Ville, Les variétés Riemanniennes de dimension $4 \frac{4}{19}$-pinceés, Ann. Inst. Fourier (Grenoble) 39 (1989), 149-154.

Department of Mathematics, University of Iowa, Iowa City, Iowa 52242 